\title{
Underpotential deposition of metals - Progress and prospects in modelling
}

\author{
V SUDHA and M V SANGARANARAYANAN* \\ Department of Chemistry, Indian Institute of Technology - Madras, Chennai 600 036, India \\ e-mail: mvs@chem.iitm.ac.in
}

MS received 28 January 2005; revised 6 May 2005

\begin{abstract}
Underpotential deposition (UPD) of metals is analysed from the perspective of phenomenological and statistical thermodynamic considerations; the parameters influencing the UPD shift have been quantitatively indicated using a general formalism. The manner in which the macroscopic properties pertaining to the depositing ions and solvent dipoles and the nature of the metallic substrate influence the UPD process are highlighted; earlier correlations of the UPD shift with the work function differences are rationalised. Anion-induced phase transitions which manifest as sharp peaks in experimental cyclic voltammograms are discussed using statistical thermodynamic models.
\end{abstract}

Keywords. Underpotential deposition; work function; surface coverage; phase transitions.

\section{Introduction}

The phenomenon of underpotential deposition (UPD) refers to the deposition of metals on foreign metal substrates at potentials that are more positive than that predicted by the Nernst equation for bulk deposition. ${ }^{1,2}$ This implies that the depositing adatoms are bound more strongly to the foreign metal electrode. Depending upon the extent of affinity with the foreign substrate, cations get reduced either partially or completely to the corresponding metal at the sub-monolayer or monolayer levels.

The mechanism of UPD has been extensively investigated with various electrochemical ${ }^{3}$ and surface characterization techniques. ${ }^{4}$ Cyclic voltammetry is customarily employed to deduce the UPD shift, viz. the difference between the peak potentials of monolayer formation and bulk deposition. ${ }^{1}$ The formation of the first monolayer is inferred from the pronounced current peaks at the potential $E>E_{\text {rev }}$ while the bulk deposition occurs at $E<E_{\text {rev }}$ where $E_{r e v}$ denotes the Nernstian equilibrium potential (for example, UPD of $\mathrm{Cu}$ on $\mathrm{Au}$ occurs at $0.46 \mathrm{~V}$ while bulk deposition occurs at $0.24 \mathrm{~V}, E_{\text {rev }}$ being $0.34 \mathrm{~V}$ vs Standard Hydrogen Electrode). Characterization techniques such as Fourier transform infrared reflection (FTIR), Surface-enhanced Raman spectroscopy

*For correspondence
(SERS), scanning tunnelling microscopy (STM), atomic force microscopy (AFM) etc. provide information regarding the surface morphology. ${ }^{5,6}$ In a complementary manner, low energy electron diffraction (LEED), X-ray photoelectron spectroscopy (XPS), Auger electron spectroscopy (AES) etc. provide more insights into the structure, energetics and composition of the various adlayers. ${ }^{7,8}$

Consistent with the development of experimental studies on UPD, density functional theory (DFT) formalism and band structure calculations have also been employed to comprehend the UPD mechanism. ${ }^{9}$ Analogously, statistical mechanical models ${ }^{10}$ and different versions dynamic Monte Carlo simulations ${ }^{11}$ have been investigated to describe the UPD vis-a-vis phase transitions. Further, the study of UPD plays a major role in the field of electrocatalysis of oxidation of organic compounds, oxygen reduction and hydrogen evolution, which are of importance in fuel cells. ${ }^{3}$ Also, the UPD modified electrodes were found to be useful to detect the intermediates formed during the reduction of aromatic nitro compounds and benzofuroxan. ${ }^{12}$ Moreover, UPD-based systems find applicability in the investigation of self assembled monolayers for developing desirable surface architectures. ${ }^{13}$

The present work essentially focuses on the different types of approaches employed for (i) computing the UPD shifts for bimetallic systems in aqueous 
and non-aqueous solvents using (a) phenomenological thermodynamics concepts, (b) density functional theories (DFT), and (iii) analysing diverse phase transitions of UPD systems with the help of equilibrium and non-equilibrium Ising model formalism.

\section{Theoretical analysis of UPD shifts}

A rigorous theoretical analysis of the underpotential deposition of metals is inherently complex, in view of the fact that the components of the system in general include the (i) metallic substrate, (ii) depositing metal ions, (iii) solvent dipoles, and (iv) adsorbed ionic species. Added to this multi-component system, is our ability to manipulate the system by an external potential (time-dependent or otherwise). Hence, it is no wonder that the first-ever correlation between the experimentally observed UPD shift and the work function difference between the substrate and the depositing metal deduced ${ }^{1}$ in 1974 has defied satisfactory explanation till now. However, without being deterred by the complexity of the problem, several noteworthy analyses have been made by the pioneering work of the groups of Rikvold, ${ }^{14,15}$ Blum, ${ }^{16}$ Leiva ${ }^{17}$ and Schmickler. ${ }^{18}$ In a way, these investigations are complimentary to one another in so far as a microscopic treatment is attempted with due mathematical, statistical mechanical and quantum mechanical sophistication.

Among several attempts to correlate the UPD shifts with the nature of the metallic substrate and depositing species, mention must be made of the attempt of Kolb et $a l^{1}$ viz.

$$
\Delta E_{\mathrm{UPD}}=0 \cdot 5\left(\Phi_{S}-\Phi_{M}\right),
$$

where $\Delta E_{\mathrm{UPD}}$ denotes the experimentally observed UPD shift and $\left(\Phi_{S}-\Phi_{M}\right)$ is the difference in the work function between the substrate and depositing species. Equation (1) is valid for most of the metal pairs, especially when polycrystalline substrates are employed. This apparently simple variation of the UPD shift is somewhat puzzling since one would anticipate a far-more involved dependence of the same, comprising the interaction energies pertaining to solvent dipoles as well as ionic species.

\subsection{Phenomenological analysis}

Since the UPD shift denotes the difference in potential between the monolayer formation and bulk deposition, it is imperative to consider the two processes separately. The monolayer formation of $\mathrm{M}$ on $\mathrm{S}$ can be envisaged as follows: ${ }^{19}$ (i) movement of the solvated ions from the bulk to the reaction zone, getting rid of their solvation sheath, and (ii) electron transfer from the substrate to the metal ions leading to the subsequent bond formation with the substrate (S). The S-M bond formation involving the defective sites ${ }^{2}$ occurs after knocking off the adsorbed solvent dipoles from the appropriate sites of the substrate. ${ }^{20}$ We note here that since the electrode is solvated, the nature of orientation of solvent dipoles at electrode surfaces also needs to be included for a rigorous analysis.

Scheme 1 depicts the constituent processes involved in the monolayer formation of $\mathrm{M}$ on $\mathrm{S}$.

Based on scheme 1, a thermochemical cycle can be constructed which leads to

$$
\Delta G_{\mathrm{ML}}=\Delta G_{\mathrm{I}}+\Delta G_{\mathrm{II}}+\Delta G_{\mathrm{III}},
$$

where $\Delta G_{\mathrm{ML}}$ denotes the Gibbs free energy change in the monolayer formation as shown in scheme 2.

In (2), $\Delta G_{\mathrm{I}}$ denotes the work done in bringing the solvated metal ions $\mathrm{M}^{n+}$ from bulk to the reaction zone represented as

$$
\Delta G_{\mathrm{I}}=W_{\mathrm{M}^{n+}},
$$

while $\Delta G_{\text {II }}$ comprises the free energy change involved in the electron transfer $\left(\Delta G_{\mathrm{et}}^{\mathrm{S}}\right)$ from the solvated electrode to the (de)solvated ions and $\Delta G^{0}$ governed by the standard reduction potential $E^{0}$ of $\mathrm{M}^{n+} / \mathrm{M}$, viz.

$$
\Delta G_{\mathrm{II}}=\Delta G_{\mathrm{et}}^{\mathrm{S}}+\Delta G^{0},
$$

$\Delta G_{\text {III }}$ involves the desorption of solvent dipoles from the defective sites of the substrate and formation of the substrate-metal $(\mathrm{S}-\mathrm{M})$ bond leading to

$$
\Delta G_{\mathrm{III}}=\theta\left(\Delta G_{\mathrm{desor}}+\Delta G_{\mathrm{S}-\mathrm{M}}^{\mathrm{inter}}\right) .
$$

The subscript/superscript "et" and "inter" denote "electron transfer" and "interfacial region" respectively. $\Delta G_{\text {desor }}$ represents the Gibbs free energy change of desorption of solvent molecules from the substrate, $\Delta G_{\mathrm{S}-\mathrm{M}}^{\mathrm{inter}}$ being the energy involved in the formation of S-M bond. $\theta$ denotes the surface coverage of the underpotentially deposited metal adatoms on the substrate. Since adsorption is visualized as the replacement of solvent molecules by the depositing species, $\theta$ is introduced in $\Delta G_{\text {desor }}$ as well as in $\Delta G_{\mathrm{S}-\mathrm{M}}^{\text {inter }}$ 


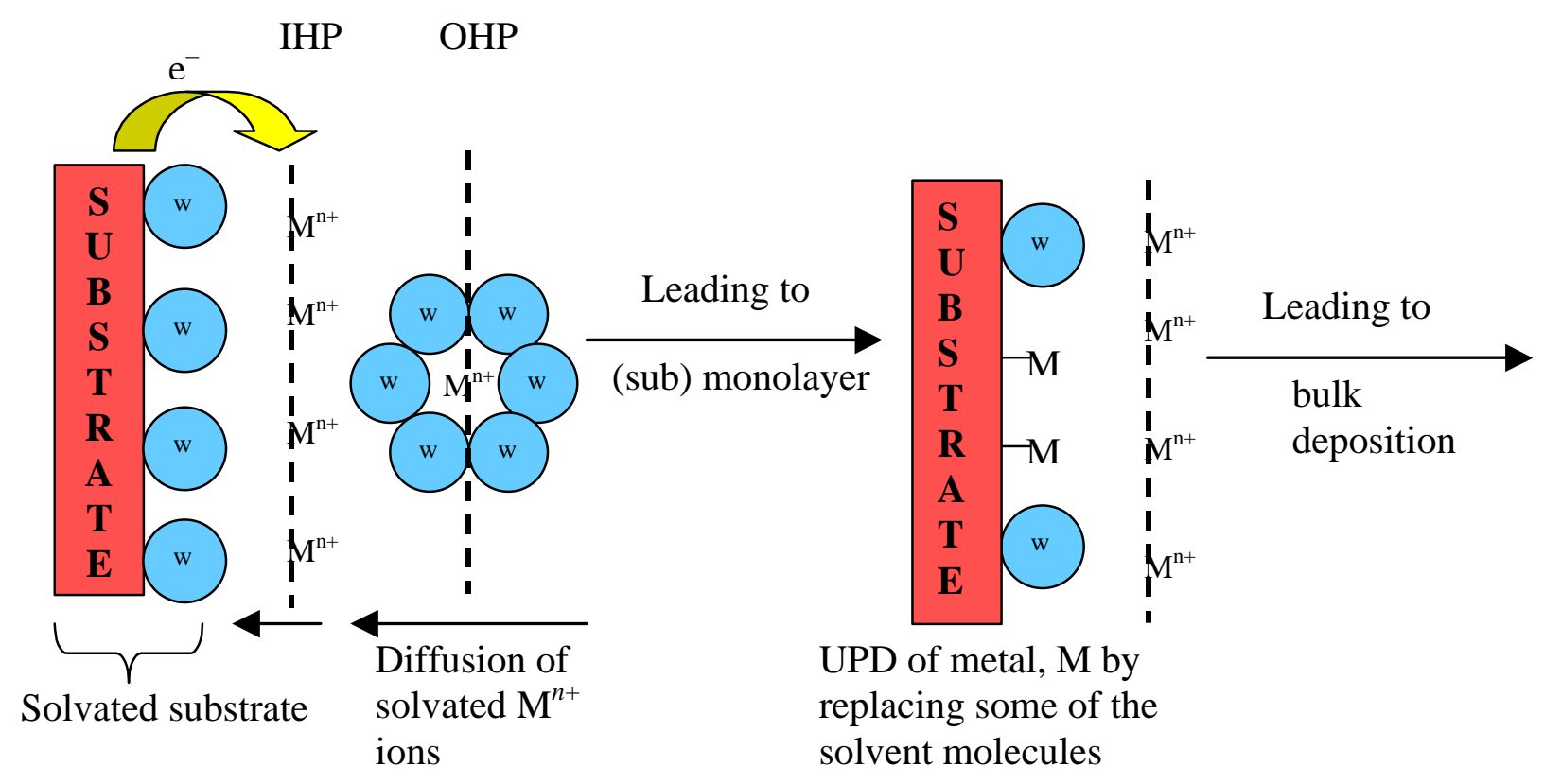

Scheme 1. Schematic representation ${ }^{19}$ of processes constituting adsorption of M upon S. IHP and OHP indicates respectively, the inner and outer Helmholtz plane. The metal ions, $\mathbf{M}^{n+}$ are surrounded by the solvent molecules ' $\mathrm{w}$ '.

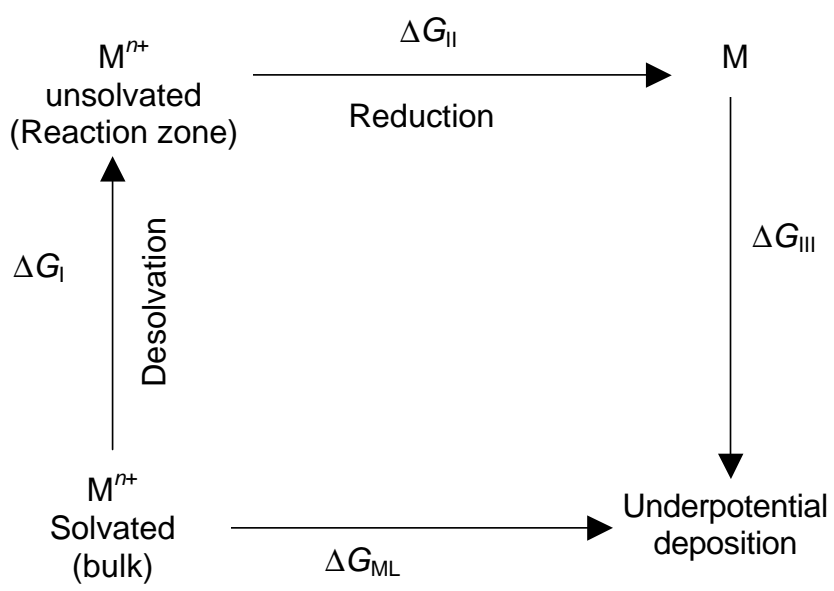

Scheme 2. Thermochemical cycle denoting different energetic contributions involved in the monolayer formation of a metal $(\mathrm{M})$ on a foreign substrate $(\mathrm{S})$.

Substituting (3) to (5) in (2), $\Delta G_{\mathrm{ML}}$ is obtained as

$$
\Delta G_{\mathrm{ML}}=W_{\mathrm{M}^{n+}}+\Delta G_{\mathrm{et}}^{\mathrm{S}}+\Delta G^{0}+\theta\left(\Delta G_{\mathrm{desor}}+\Delta G_{\mathrm{S}-\mathrm{M}}^{\mathrm{inter}}\right) .
$$

While the analysis so far has been general, we now indicate its applicability to predict the UPD shifts of aqueous solvents.

2.1a Aqueous solvents: The terms $W_{\mathrm{M}^{n+}}, \Delta G_{\mathrm{et}}^{\mathrm{S}}$ and $\Delta G_{\text {desor }}$ involve the solvent characteristics. To com- pute these, the formulation of the work function of hydrated electrodes as well as the energetics involved in the binding of water molecules becomes essential. By invoking the classification of metals into $s p$ and $d$-blocks and introducing the dipole potential barrier, surface potential of electrons in solution $^{21}$ etc., the potential of the monolayer adsorption follows as ${ }^{19}$

$$
\begin{aligned}
E_{\mathrm{ML}} & =-\frac{\Delta G_{\mathrm{desolv}}}{n F S_{N}}-\frac{\chi_{\mathrm{M}^{n+}}}{n}+\frac{\Phi_{S}}{C N_{S}} \\
& +E^{0}-\frac{\theta \Delta G_{\mathrm{S}-\mathrm{H}_{2} \mathrm{O}}}{n F}-\frac{\theta \Delta H_{\mathrm{S}-\mathrm{M}}}{n F C N_{S}},
\end{aligned}
$$

where $\Delta G_{\text {desolv }}$ is the bulk dehydration energy of the (depositing) metal ions, $S_{N}$ being the hydration number, $n$ denotes the number of electrons involved in charge transfer and $\chi_{\mathrm{M}^{n+}}$ indicates the surface potential of electrons in metal, $C N_{S}$ and $\Phi_{S}$ represent the lattice coordination number and work function of the substrate respectively. The terms $\Delta G_{\mathrm{S}_{-} \mathrm{H}_{2} \mathrm{O}}$ and $\Delta H_{\mathrm{S}-\mathrm{M}}$ denote respectively the Gibbs free energy change involved in the substrate-water bond formation and enthalpy change of substratemetal bond formation available in tabular compilations. By approximating $\chi_{e}^{M}$ as $^{21}-0.4 \mathrm{eV}$ and taking other tabulated parameters such as work functions, 
Table 1. Estimation of the UPD shift using (8).

\begin{tabular}{|c|c|c|c|c|c|c|c|c|}
\hline System & $\begin{array}{l}\text { Work func. } \\
\text { of the } \\
\text { substrate }^{21} \\
\Phi_{S}(\mathrm{eV})\end{array}$ & $\begin{array}{c}\text { Work func. } \\
\text { of the } \\
\text { ads. }^{21} \\
\Phi_{M}(\mathrm{eV})\end{array}$ & $\begin{array}{l}\text { Charge } \\
\text { on the } \\
\text { ads. }{ }^{19} \\
z_{a d}\end{array}$ & $\begin{array}{c}\text { Surface } \\
\text { coverage }^{2} \\
\theta\end{array}$ & $\begin{array}{c}\text { Coord. } \\
\text { number } \\
\text { of the } \\
\text { substrate }^{86} \\
\quad C N_{S}\end{array}$ & $\begin{array}{l}\text { Coord. } \\
\text { number } \\
\text { of the } \\
\text { ads. }^{86} \\
C N_{M}\end{array}$ & $\begin{array}{c}\text { Calc. } \\
\text { UPD } \\
\text { shift } \\
\Delta E_{\mathrm{UPD}} \\
(\text { calc })(\mathrm{V})\end{array}$ & $\begin{array}{c}\text { Experi- } \\
\text { mental } \\
\text { UPD shift }^{2} \\
\Delta E_{\mathrm{UPD}} \\
(\operatorname{expt})(\mathrm{V})\end{array}$ \\
\hline $\mathrm{Au} / \mathrm{Cu}^{2+}$ & $4 \cdot 80$ & $4 \cdot 55$ & 2.00 & $0 \cdot 18$ & $5 \cdot 56$ & $5 \cdot 56$ & $0 \cdot 18$ & $0 \cdot 22$ \\
\hline $\mathrm{Pt} / \mathrm{Cu}^{2+}$ & 5.03 & 4.55 & 1.00 & 0.23 & 6.00 & $5 \cdot 56$ & 0.43 & 0.44 \\
\hline $\mathrm{Ag} / \mathrm{Tl}^{+}$ & $4 \cdot 30$ & $4 \cdot 10$ & $3 \cdot 53$ & $0 \cdot 22$ & $5 \cdot 56$ & $3 \cdot 56$ & 0.25 & $0 \cdot 28$ \\
\hline $\mathrm{Ag} / \mathrm{Cd}^{2+}$ & $4 \cdot 30$ & $4 \cdot 12$ & 2.95 & $0 \cdot 17$ & $5 \cdot 56$ & $4 \cdot 56$ & $0 \cdot 16$ & $0 \cdot 16$ \\
\hline $\mathrm{Ag} / \mathrm{Pb}^{2+}$ & $4 \cdot 30$ & $4 \cdot 24$ & $4 \cdot 16$ & $0 \cdot 10$ & $5 \cdot 56$ & 2.56 & $0 \cdot 12$ & $0 \cdot 16$ \\
\hline $\mathrm{Cu} / \mathrm{Tl}^{+}$ & 4.55 & $4 \cdot 10$ & 2.76 & 0.31 & 5.56 & 3.56 & 0.34 & 0.34 \\
\hline $\mathrm{Au} / \mathrm{Pb}^{2+}$ & $4 \cdot 80$ & $4 \cdot 24$ & $1 \cdot 50$ & 0.22 & $5 \cdot 56$ & $2 \cdot 56$ & 0.40 & 0.40 \\
\hline
\end{tabular}

Abbreviations: func. - function; ads. - adsorbate; Coord. - coordination; Calc. - calculated

lattice coordination numbers etc. $E_{\mathrm{ML}}$ can be estimated for a chosen system.

In an analogous manner, the potential for bulk deposition can be formulated mutatis mutandis yielding the UPD shift as

$$
\begin{aligned}
\Delta E_{\mathrm{UPD}}= & \theta\left(\frac{\Phi_{S}}{C N_{S}}-\frac{\Phi_{M}}{C N_{M}}\right)+\frac{(1-2 \theta) \Delta G_{\mathrm{S}-\mathrm{H}_{2} \mathrm{O}}}{z_{a d} F} \\
& +\frac{(1-2 \theta) \Delta H_{\mathrm{S}-\mathrm{M}}}{z_{a d} F C N_{S}}+\frac{\theta \Delta G_{\mathrm{M}-\mathrm{H}_{2} \mathrm{O}}}{z_{a d} F}+\frac{\theta \Delta H_{\mathrm{M}-\mathrm{M}}}{z_{a d} F C N_{M}} .
\end{aligned}
$$

In view of the partial charge transfer between the depositing species and the metal, the charge on the adsorbate $z_{a d}$ arises instead of $n$-the number of electrons involved in the reduction process. A comparison with (7) reveals the presence of the coordination number of the depositing metal $\left(C N_{M}\right)$, its work function $\left(\Phi_{\mathrm{M}}\right)$ as well as the energetics involved in $\mathrm{M}-\mathrm{H}_{2} \mathrm{O}$ and $\mathrm{M}-\mathrm{M}$ bond formation. In table 1, the UPD shifts for a few systems estimated from (8) are compared with the experimental data. Although the UPD shifts of nearly forty systems have been experimentally reported, we have restricted ourselves here to a smaller subset in view of the necessity for employing precise values of partial charge transfer, number of monolayers constituting the bulk deposition etc. in (8)

Although (8) provides a preliminary methodology for evaluating the UPD shifts, it offers a framework on the basis of which further refinements can be accomplished. The correlation of the UPD shifts with work function differences inferred by Kolb ${ }^{2}$ may essentially be ascribed to the interplay between the magnitude of various competing factors appearing in (8). The fact that incorporation of work function differences alone are insufficient to interpret the
UPD shift has been pointed out more than two decades $\mathrm{ago}^{22}$ and the influence of structural parameters in rationalizing the UPD shift has also been indicated. Moreover, this correlation has been explained by Leiva, ${ }^{23}$ in terms of a thermodynamic derivation involving the adsorption energies of substrate and adsorbate.

The present phenomenological formalism also provides an insight into the various system parameters which influence $\theta$ vis-a-vis UPD shift. Using (8), we obtain

$$
\Delta E_{\mathrm{UPD}}=A+B \theta
$$

where the parameters $A$ and $B$ are as follows:

$$
A=\Delta H_{\mathrm{S}-\mathrm{M}} / z_{a d} F C N_{S}+\Delta G_{{\mathrm{S}-\mathrm{H}_{2} \mathrm{O}}} / z_{a d} F
$$

and

$$
\begin{aligned}
B & =\Phi_{S} / C N_{S}-\Phi_{M} / C N_{\mathrm{M}}-2 \Delta G_{\mathrm{S}_{-} \mathrm{H}_{2} \mathrm{O}} / z_{a d} F \\
& +\Delta G_{\mathrm{M}-\mathrm{H}_{2} \mathrm{O}} / z_{a d} F-2 \Delta H_{\mathrm{S}-\mathrm{M}} / z_{a d} F C N_{S} \\
& +\Delta H_{\mathrm{M}-\mathrm{M}} / z_{a d} F C N_{M} .
\end{aligned}
$$

The variation of $\Delta E_{\text {UPD }}$ with $\theta$ is linear. Further, the intercept is composed of the free energy change involved in the desorption of water molecules from the substrate and enthalpy changes. However, the slope is crucially dependent upon work function differences as well as enthalpies of $\mathrm{S}-\mathrm{M}$ and $\mathrm{M}-\mathrm{M}$ bonds for a chosen solvent. Takayanagi et $a l^{24}$ described the various structures of $\mathrm{Pb}$ formed for different coverage on $\operatorname{Ag}(111)$ during the process of UPD using LEED patterns, AES analysis and reflectance measurements. Further, the variation of work function with surface coverage of the adsorbate is a topic of much interest in density functional theories of chemisorption. 
2.1b Non-aqueous solvents: Among various nonaqueous solvents, acetonitrile (ACN) and propylene carbonate (PC) are widely employed, the depositing species in general being alkali metals. Hence, the estimation of the UPD shift pertaining to the alkali metals in the above solvents has been recently investigated. ${ }^{25}$ In contrast to the earlier analysis pertaining to aqueous solvents, the potential half-width $\left(\Delta V_{1 / 2}\right)$ data have been employed in this case for estimating the surface coverages. By employing the Frumkin isotherm in which the half-width potential, $\Delta V_{1 / 2}$ is related to the interaction constant $a$ as, ${ }^{26}$

$$
\begin{aligned}
\Delta V_{1 / 2}= & \frac{2 R T}{F} \ln \left[\frac{(a+4)^{1 / 2}+(a+1)^{1 / 2}}{(a+4)^{1 / 2}-(a+1)^{1 / 2}}\right] \\
& +\frac{2 a R T}{n F}\left[\frac{(a+1)}{(a+4)}\right]^{1 / 2} .
\end{aligned}
$$

$a$ is evaluated which in turn yields the charge of the adsorbate, $q_{p}$. Consequently, the surface coverage, ( $\left.\theta=q_{p} / q_{\text {max }}\right)$ can be calculated by knowing the charge density associated with maximum surface excess, $q_{\max }$. The bonding pattern of ACN and PC are inferred from spectroscopic and surface characterization studies. ${ }^{27-30} \mathrm{UPD}$ shifts of $\mathrm{Au} / \mathrm{Li}^{+}(\mathrm{ACN})$, $\mathrm{Cu} / \mathrm{Li}^{+}$(PC), $\mathrm{Pt} / \mathrm{Li}^{+}$(PC), $\mathrm{Pt} / \mathrm{Na}^{+}$(PC) and $\mathrm{Pt} / \mathrm{Cs}^{+}$ (PC) have been computed which show satisfactory agreement with the experimental data.

\subsection{Density functional theories for estimating the UPD shifts}

In view of the success of the density functional theories (DFT) for elucidating the structure of the metal/electrolyte interfaces, ${ }^{31,32}$ it is anticipated that DFT should play a prominent role in interpreting the UPD shifts of various single crystal (and polycrystalline) electrodes. In this respect, DFT is particularly appealing in so far as (i) electronic density of metals can be incorporated from the beginning, and (ii) first principles calculation of the work function changes with adsorbate coverages, is feasible.

However, several bottlenecks arise while transcribing the DFT of metal/electrolyte interfaces to UPD phenomena. For example, in contrast to the electrical double layer theories, the description of UPD involves two different metals as well as detailed structural features of the substrate along with the inter-metallic interactions. Consequently, extensive studies regarding the applicability of DFT to
UPD are still lacking, except for a few studies. ${ }^{9,17,33,34}$

DFT envisages formulating a functional for energy in terms of the appropriate electron densities. ${ }^{35,36}$ In the widely employed jellium model, the positive charge of the metal lattice is smeared out and uniformly distributed throughout the space up to the metal surface, where it drops abruptly to zero, the conduction electrons being allowed to spill over beyond the surface. ${ }^{33}$ In a general manner, $E[n(x)]$ is given by

$$
E[n(x)]=E_{\mathrm{k}}+E_{\mathrm{xc}}+E_{\mathrm{ps}}+E_{\mathrm{el}}
$$

where $E_{\mathrm{k}}, E_{\mathrm{xc}}, E_{\mathrm{ps}}$ and $E_{\mathrm{el}}$ denote the kinetic, exchange and correlation, pseudopotential and electrostatic energies respectively. Subsequently, the electron density profile $n(x)$ is written in terms of the "spill-over" distance. In the original version" of DFT, $n(x)$ is given by,

$$
n(x)=n_{e l}\left(1-\frac{1}{2} e^{\alpha x}\right) \theta(-x)+\frac{1}{2} n_{e l} e^{-\alpha x} \theta(x),
$$

where $n_{e l}$ is the bulk electron density of the metal, $\alpha$ denotes the free parameter measuring the extent of electronic spill over into the exterior and $\theta(x)$ is the Heaviside step function. A very lucid self-consistent analysis then yields the work function, dipole barrier etc. albeit for the metal/vacuum interface. ${ }^{9}$ Several refinements with respect to various terms of (13) and (14) have been extensively discussed ${ }^{10,38}$ and partially implemented for electrochemical contexts also. ${ }^{39}$

Since the properties of metals are dependent to a greater extent on the valence electrons rather than on the tightly bound core electrons, the pseudopotential approximation is invoked. The pseudopotential is constructed such that the scattering properties of the pseudo wavefunctions become identical to the scattering properties of the ion and core electrons. Among several pseudopotential prescriptions, the Ashcroft pseudopotential is especially convenient, in view of its simplicity. The Ashcroft pseudopotential, $w(r)$ for the interaction between an ion of charge $z$ and an electron at $r$ is represented as ${ }^{40}$

$$
\begin{aligned}
w(r) & =0, & & \text { if } r<r_{c} \\
& =-z / r, & & \text { if } r \geq r_{c}
\end{aligned}
$$

where $r_{c}$ denotes the pseudopotential radius of the adsorbate. 
The underpotential deposition shift is written as,

$$
\Delta E_{\mathrm{UPD}}=\left[E_{\mathrm{bind}}(S / M)-E_{\mathrm{bind}}(M / M)\right] / z,
$$

where $E_{\text {bind }}(S / M)$ denotes the binding energy of the adsorbate on the substrate and $E_{\text {bind }}(M / M)$ represents the binding energy of the adsorbate on the adsorbate. Although several methods exist for choosing $r_{c}$, Schmickler ${ }^{32}$ employs a minimization approach viz.

$$
\mathrm{d} E_{\text {bind }}(M / M) / \mathrm{d} r_{s}=0,
$$

leading to the pseudopotential radius $r_{c}$ as

$$
\begin{aligned}
r_{c}=\frac{1}{3}\left(-4.42 r_{s}+0.9162 r_{s}^{2}\right. & \\
& \left.+\frac{0 \cdot 878 r_{s}^{4}}{\left(r_{s}+7 / 0 \cdot 8\right)^{2}}-M z^{2 / 3} r_{s}^{2}\right)^{1 / 2},
\end{aligned}
$$

where $r_{s}=\left(3 / 4 \pi n_{e l}\right)^{1 / 3}$ is the Wigner-Seitz radius of the electron gas, $M$ being the Madelung constant appropriate for the lattice. Hence, using (17) and (18), $E_{\text {bind }}(M / M)$ is derived as

$$
\begin{aligned}
& E_{\text {bind }}(M / M)= \\
& \quad z\left(\frac{1 \cdot 105}{r_{s}^{2}}-\frac{0.4581}{r_{s}}-\frac{0.439}{r_{s}+7.8}+M \frac{z^{2 / 3}}{2 r_{s}}+2 \pi n_{e l} r_{c}^{2}\right)
\end{aligned}
$$

where the terms in parentheses denote respectively the kinetic, exchange, correlation, electrostatic and pseudopotential energy per electron.

While applying (19) for the evaluation of $E_{\text {bind }}(S / M)$, the electrostatic energy term needs to be rewritten incorporating (i) the electrostatic selfinteraction of the adsorbate layer for various adsorbate structures, $E_{\mathrm{el}}^{2 d}$; (ii) the electrostatic interaction of the adsorbate layer with the substrate, $E_{\mathrm{el}}^{\mathrm{int}}$ and (iii) the difference between the electronic surface relaxation energy of the substrate-adsorbate system and that of the pure substrate, $\Delta E_{s}$. A detailed analysis of the above mentioned terms incorporating the nature of the substrate along with the structure of the adsorbed layer has been provided by Leiva. ${ }^{9}$ With the help of (16), the UPD shifts of metals Li, $\mathrm{Na}, \mathrm{Zn}, \mathrm{Cd}, \mathrm{Tl}$ and $\mathrm{Pb}$ on polycrystalline and single crystal surfaces of Al have been estimated. ${ }^{41}$ Subse- quently, the methodology has been extended to the systems $\mathrm{Tl}$ and $\mathrm{Pb}$ UPD on $\mathrm{Ag}(111),{ }^{38} \mathrm{Cu}$ UPD on $\mathrm{Ag}(111),{ }^{34} \mathrm{Ag}$ on $\mathrm{Au}(111),{ }^{34} \mathrm{Cu}$ on $\mathrm{Au}(100)$ and $\mathrm{Au}(111)^{17}$ employing sophisticated DFT-based computations. The energies of adsorbed hydrogen and $\mathrm{CO}$ at thin $\mathrm{Pd}$ films on $\mathrm{Au}(111)$ and $\mathrm{Au}(100)$ have also been estimated using this methodology. ${ }^{42}$ Although the above systems illustrate the potentiality of DFT-based approaches for interpreting the UPD shifts involving single crystal electrodes, quantitative agreement with experimental results is still lacking. In addition, this methodology does not incorporate the explicit influence of solvent dipoles.

\section{Phase transitions involving UPD systems}

A variety of phase transitions exist at electrode/ electrolyte interfaces including orientational orderdisorder transitions, ${ }^{43}$ surface crystallization ${ }^{44}$ and two-dimensional condensation. ${ }^{45}$ Underpotential deposition of metals constitutes an illustrative example for elucidating various aspects of phase transitions at electrochemical interfaces in so far as (i) surface coverages, (ii) coulombic and noncoulombic interactions, (iii) chemical potentials, (iv) applied electric field, and (v) structural features of the substrate arise during the formulation of the appropriate Hamiltonian. Hence, various strategies pertaining to Ising or equivalently lattice gas models have been extensively employed in this context with a view to obtaining new insights.

\subsection{Equilibrium Ising models}

The Ising model has been originally proposed for describing phase transitions in magnetic systems. ${ }^{46}$ Its equivalence with lattice gas models is especially attractive since it enables transcription of results from the magnetic literature to condensed matter physics. The spin $1 / 2$ and spin 1 generalized Ising model formalism for describing adsorption at electrochemical interfaces in an unified manner has been advocated. ${ }^{47,48}$ The mean field analysis of twodimensional condensation pertaining to adsorption of organic compounds at electrode/electrolyte interfaces employing spin $1 / 2$ Ising model formalism in order to deduce the critical temperatures and critical exponents has also been proposed. ${ }^{49}$ The possibility of constructing spin-1 (three state) phase diagrams for competitive adsorption of two different dipoles has been indicated earlier. ${ }^{48}$ 


\subsection{Kinetic Ising model versions}

In contrast to the comprehensive literature on the applicability of equilibrium Ising models for studying electrosorption vis-a-vis electrochemical phase transitions, the study of non-equilibrium (or equivalently kinetic) Ising models has been less extensive. Interestingly, the isomorphism of the kinetic Ising model formalism with phenomenological transport equations was first demonstrated (somewhat unexpectedly!) for electron hopping in redox polymer electrodes. ${ }^{50,51}$ However, the most extensive application of kinetic (non-equilibrium) Ising models in electrochemistry has been carried out by the group of Rikvold ${ }^{15}$ for diverse situations viz. adsorption of chlorides and bromides on $\mathrm{Ag}(100)$, electrodeposition of urea on $\mathrm{Pt}(100)$ and UPD of $\mathrm{Cu}$ on $\mathrm{Au}$ (111). In these investigations, the crucial step consists in the formulation of the appropriate Hamiltonian with nearest (and next nearest) neighbour interaction energies in conjunction with the appropriate prescription for the Monte Carlo step.

The kinetic Ising model postulates the timeevolution of a chosen site via a generalized master equation $(\mathrm{GME})$ as $^{51}$

$$
\begin{aligned}
\frac{\partial}{\partial t} P_{N}\left(\{\sigma\}_{N} ; t\right) & =\sum_{\langle i j\rangle} W_{i j}\left(\sigma_{i} \sigma\right\}_{j}\{\sigma) P_{N}\left(\{\sigma\}_{N}^{i j} ; t\right) \\
& -\sum_{\langle i j\rangle} W_{i j}\left(\sigma_{j} \sigma_{i}\{\sigma\}_{i j}\right) P_{N}\left(\{\sigma\}_{N} ; t\right) \\
& +\sum_{\langle j k\rangle} W_{j k}\left(\sigma_{k} \sigma_{j}\{\sigma\}_{j k}\right) P_{N}\left(\{\sigma\}_{N}^{j k} ; t\right) \\
& -\sum_{\langle j k\rangle} W_{j k}\left(\sigma_{j} \sigma_{k}\{\sigma\}_{j k}\right) P_{N}\left(\{\sigma\}_{N} ; t\right),
\end{aligned}
$$

where $P_{N}\left(\{\sigma\}_{N} ; t\right)$ denotes the normalized $N$ spin distribution function at $t .\{\sigma\}_{N}^{i j}$ and $\{\sigma\}_{N}^{i k}$ are configurations wherein $\sigma_{i} \sigma_{j} ; \sigma_{j} \sigma_{k}$ have interchanged their spin configurations with that of $\{\sigma\}_{N} ; W_{i j}$ and $W_{j k}$ represent the transition probabilities. The Hamiltonian formulated in a suitable ensemble then leads to the partition function, wherefrom other thermodynamic quantities can be derived. The transition probabilities are represented in terms of either Glauber dynamics ${ }^{52}$ (time-dependent adsorption problems) or Kawasaki dynamics ${ }^{53}$ (charge transport processes). The GME as shown above is too detailed for further analysis; hence the focus has essentially been on the reduced master equation containing the expectation value of the random variables, viz. $\left\langle\sigma_{i}\right\rangle$.
The ensemble average of the pseudo-spin variable $\left(\sigma_{i}\right)$ is then related to the experimental observables viz. current/electron diffusion coefficient in the case of charge transport and surface coverage for adsorption processes. Scheme 3 illustrates the methodology involved in the GME approach to electrochemical studies.

In order to apply the kinetic Ising Model approaches to UPD phenomena, it is imperative to formulate the appropriate GME's. Subsequently, three options suggest themselves, viz. (i) conversion of these discrete descriptions to continuum versions, (ii) numerical solution of the spatio-temporal transport equations pertaining to the situation under consideration, and (iii) Monte Carlo simulations to deduce the system response. While the conversion of the 'discrete' description to continuum versions such as the Nernst-Planck equation for diffusionmigration and Saveant equation for electron hopping in redox polymer electrodes have been demonstrated, ${ }^{50,51}$ similar hierarchical descriptions pertaining to UPD are still lacking.

The numerical solution of the spatio-temporal transport equations can be employed in various situations such as time evolution of surface cover$\operatorname{ages}^{54}$ and theoretical simulation of cyclic voltam-

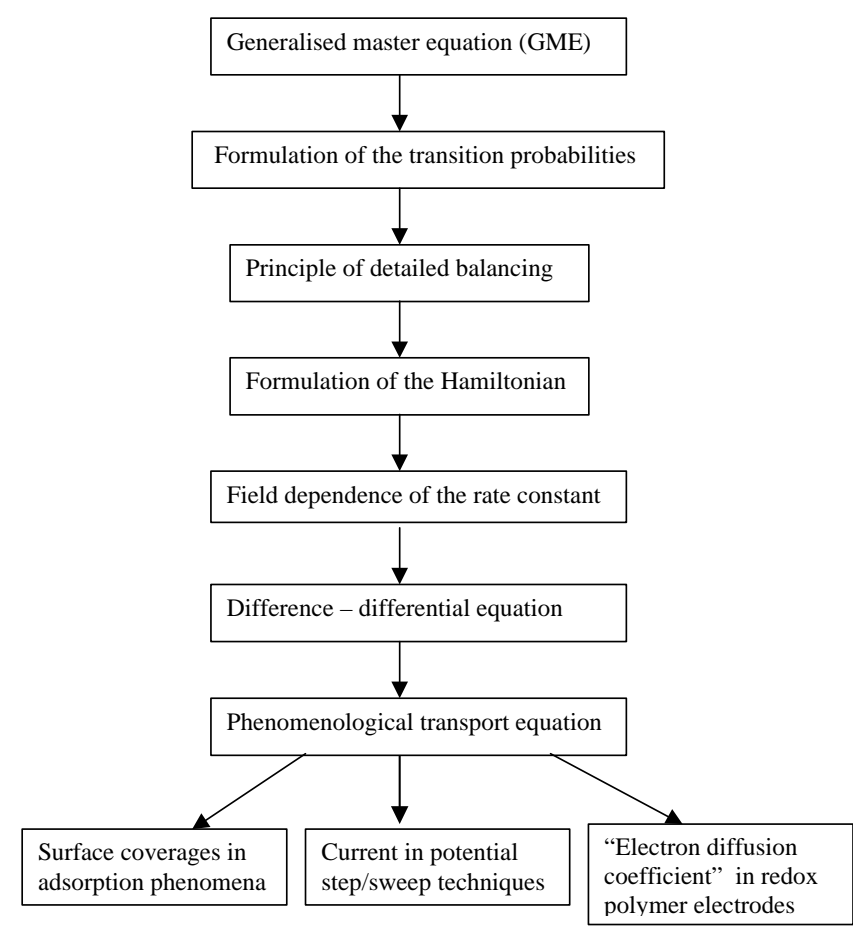

Scheme 3. Steps involved in the analysis of GME for electrochemical systems. 
mograms/potential step responses. ${ }^{55}$ The applicability of diverse Monte Carlo simulation procedures to a variety of interesting issues in electrosorption and underpotential deposition has been demonstrated by Rikvold et $a l^{56}$ and Leiva et $a l .^{57}$ In particular, the construction of the ground-state phase diagrams and the rules governing the transition rates in a microscopic description need a careful analysis, lest erroneous conclusions emerge. ${ }^{58}$

\subsection{Phase transitions and underpotential deposition of metals}

The exact solution of the two-dimensional IsingHamiltonian in a zero magnetic field is originally due to Onsager ${ }^{59}$ in 1944. Although an exact solution for non-zero magnetic field has been elusive till now, methods employing series expansions, renormalization group $^{60}$ and related techniques ${ }^{61}$ have provided solutions which may be termed as "accurate". In this instance, the two-point Padé approximant $^{62}$ is especially elegant since it provides a single expression valid for the entire regime after combining the expressions derived in different domains*. The efficacy of Padé approximants in the study of $\mathrm{Cu}$ on $\mathrm{Au}(111)$ has been illustrated by Blum et al. ${ }^{63}$

The UPD system of $\mathrm{Cu}$ on $\mathrm{Au}(111)$ has been discussed using a simplified spin-1 Ising model represented by Blume-Emery-Griffiths (BEG) Hamiltonian. ${ }^{64}$

We may note that the BEG Hamiltonian studied by Tarasenko et $a l^{65}$ in this context is a particular case of the generalised spin-1 Hamiltonian for a ternary system. ${ }^{66}$ In the case of the UPD analysis, a three-state (spin-1) Hamiltonian can be formulated incorporating the vacancies, cations and anions. By defining current response as

$$
I(\Psi)=\frac{\partial \theta}{\partial \Psi} \frac{\mathrm{d} \Psi}{\mathrm{d} t}
$$

where $\theta$ denotes the surface coverage of the depositing species and $\Psi$ indicates the potential bias, it is possible to theoretically construct cyclic voltammetric response of copper UPD on $\mathrm{Au}(111)$ in presence of adsorbed sulphate ions. Figure 1 depicts the

\footnotetext{
*The Padé approximants have been demonstrated to be valuable for studying diffusion at ultramicroelectrodes ${ }^{68}$ cyclic voltammetric current response ${ }^{69}$ and potential transients for electron transfer process coupled with chemical reactions ${ }^{70}$
}

simulated CV profiles wherein two distinct peaks at $185 \mathrm{mV}$ and $265 \mathrm{mV}$ are noticed, ${ }^{15}$ while the $\mathrm{CV}$ experimental data ${ }^{67}$ results show that the peaks are separated by $\sim 50-150 \mathrm{mV}$ depending upon the electrolyte concentrations and scan rates.

The UPD of $\mathrm{Cu}$ on $\mathrm{Au}(111)$ in presence of $\mathrm{SO}_{4}^{2-}$ is visualized by ${ }^{71}$ Huckaby and Blum as shown in figure 2 , which results in the formation of a $\sqrt{3} \times \sqrt{3}$ film of sulphate ions by occupying one of the three triangular sublattices. Each adsorbed sulphate ion is represented by a set of three lines centred at the adsorption site and ending at the three associated gold atoms. Each line represents an $\mathrm{S}-\mathrm{O}$ bond. Then the copper ions get adsorbed on the free adsorption sites (sticky sites), forming a two-dimensional honeycomb lattice. Based on this, a statistical mechanical

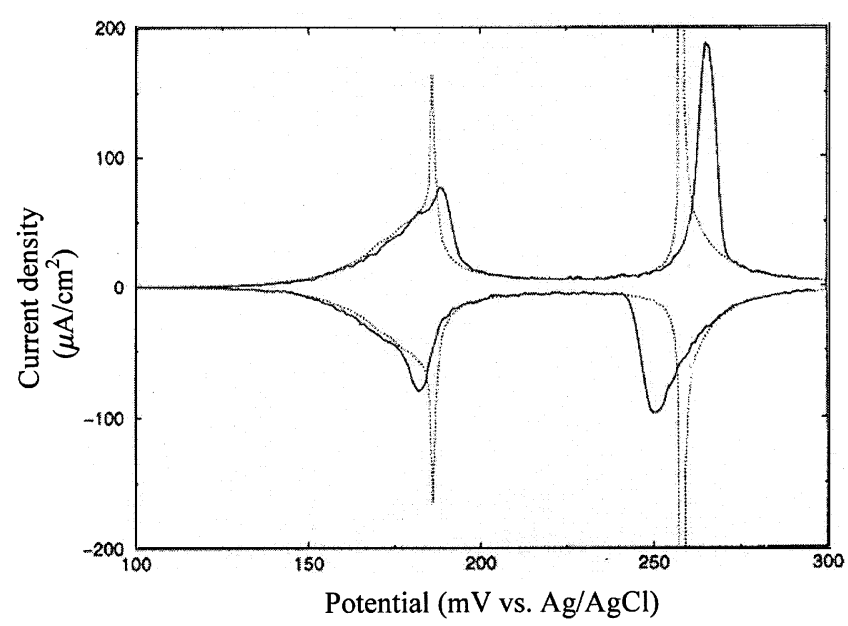

Figure 1. Simulated CV profiles for $\mathrm{Cu}$ UPD on $\mathrm{Au}(111)$. Solid lines represent the molecular dynamics simulation results with a scan rate of $10 \mathrm{mV} / \mathrm{s}$, while dotted lines denote the quasi-equilibrium estimates. ${ }^{15}$

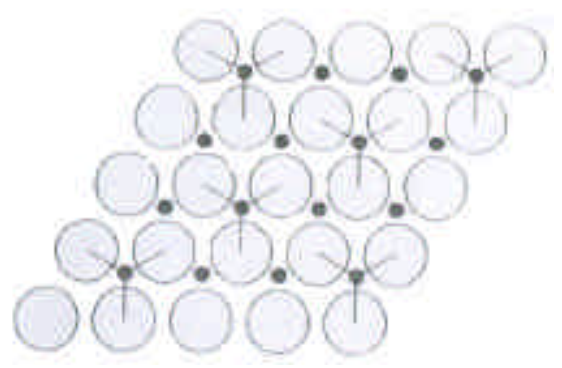

Figure 2. A schematic description of the ordered $\sqrt{3} \times$ $\sqrt{3}$ sulphate phase on $\mathrm{Au}(111)$. $\mathrm{Au}$ atoms are represented by large spheres while the adsorption sites for both $\mathrm{SO}_{4}^{2-}$ and $\mathrm{Cu}$ are depicted as black spheres. The adsorbed $\mathrm{SO}_{4}^{2-}$ groups are depicted as sets of three lines emerging from the adsorption sites to the neighbouring Au atoms. ${ }^{71}$ 
model in conjunction with the fugacity expansions arising from the Padé approximants is proposed yielding the theoretical cyclic voltammogram shown ${ }^{71}$ in figure 3 in gross agreement with the experimental observation of two sharp spikes separated by a potential range of $150 \mathrm{mV}$ with the relative area of the two spikes being in the ratio $1: 2$.

It must be pointed out here that on account of the versatility of the (single crystal and polycrystalline) substrates and diversity of electrolytes customarily employed, UPD systems offer a fertile ground for applying the sophisticated statistical mechanical models pertaining to various types of lattices (square, triangular, honeycomb, hard hexagon etc.) and diverse interaction schemes.

\subsection{Cluster variation method for the UPD of $\mathrm{Cu}$ on $\mathrm{Au}(111)$}

The limitations of the Bragg-Williams (or equivalently mean field) approximation in the study of critical phenomena have been well-documented in the literature. $^{72}$ Consequently, hierarchical improvements have been sought for solving the two(and three-) dimensional Ising-Hamiltonians pertaining to various lattices. Among these refinements, mention may be made of the following: (i) Bethe (quasichemical) approximation, ${ }^{73}$ (ii) cluster variation method, ${ }^{74}$ and (iii) renormalisation group. ${ }^{60} \mathrm{In}$

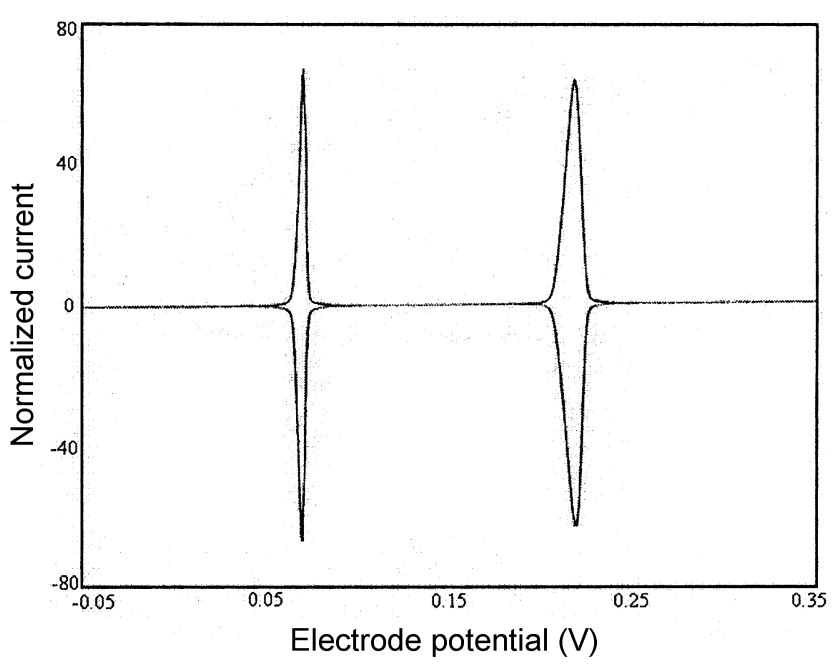

Figure 3. Theoretical cyclic voltammogram with two sharp peaks corresponding to two first-order phase transitions for UPD of $\mathrm{Cu}$ on $\mathrm{Au}(111)$ in the presence of sulphate $^{71}$. the context of electrical double layer theories, the Bethe approximation has been employed for interfacial solvent structure using a two-state solvent model ${ }^{75}$ and adsorption of organic compounds, ${ }^{76}$ while a variant of the approximation (ii) has been studied by Schmickler ${ }^{77}$ for water dipoles adsorbed at metal electrodes.

In the simple mean field approximation of Ising models, each spin is treated to be statistically independent of all the other spins, viz. statistical correlation effects are ignored. A more refined approximation consists in analysing a cluster of spins and handling the interactions among the spins within the cluster exactly and the interactions of spins outside of the cluster as in a mean-field approximation. The cluster variation method was first invoked by Kikuchi while analysing the magnetic properties. ${ }^{74}$ The mean field approximation consists in neglecting all the cumulant functions except the first cumulant arising from the first cluster function. Analogously, the Bethe approximation invokes the two-site or pair-approximation. It is possible to consider large cluster sizes and, as anticipated, the numerical analysis becomes more involved, with the chosen size of the cluster dictating the complexity of the nonlinear equations to be solved.

Huckaby et $a l^{78}$ have applied the cluster variation method for the UPD of $\mathrm{Cu}$ on $\mathrm{Au}(111)$ in the presence of bisulphate ions. The Hamiltonian is formulated for a triangular lattice assuming that a given site is (i) either vacant or (ii) occupied by a bisulphate ion or copper adatom. The Helmholtz free energy is minimized for obtaining the adsorption isotherms and equations of state. The discontinuities in the surface coverages which signify the onset of phase transitions are reflected as spikes in the experimental cyclic voltammograms for this system. ${ }^{79,80}$ In order to apply the method to UPD of $\mathrm{Cu}$ on $\mathrm{Au}(111)$ in the presence of bisulphate, clusters containing six adsorption sites were chosen. The two order-disorder transitions were deduced from the discontinuities in the adsorption isotherms pertaining to bisulphate ions and copper. These two phase transitions in fact correspond to the two spikes in the experimental cyclic voltammograms, ${ }^{81}$ thereby proving the effectiveness of the cluster variation method for phase transitions involving UPD systems.

Although the above discussion of phase transitions in UPD systems has been confined to the system of $\mathrm{Cu}$ on $\mathrm{Au}(111)$ in the presence of sulphate and bisulphate ions, the field of anion-induced 
phase transitions has been a topic of intense research activity during the past decade, from statistical thermodynamic perspective as well as spectroscopic and surface characterization studies. Other anions such as chloride, bromide and perchlorate have also been studied on diverse single crystals of $\mathrm{Ag}, \mathrm{Au}$ and $\mathrm{Pt}$ with different depositing species $\mathrm{Cu}$, $\mathrm{Cd}, \mathrm{Pb}, \mathrm{Bi}, \mathrm{Zn}, \mathrm{Tl}$ etc. In this context, infrared spectroscopy and scanning tunnelling microscopy in conjunction with radiotracer studies have revealed interesting surface morphologies of adlayers mediated by the specificity of anions. ${ }^{82,83}$ The theoretical analysis of these multi-component phase diagrams, however, has lagged behind.

\section{Perspectives and summary}

The foregoing critique illustrates subtle features involved in the theoretical analysis of UPD employing phenomenological and microscopic theories. Issues such as (i) estimation of the UPD shifts from the system parameters pertaining to ions, solvent dipoles and metals, (ii) efficacy of the DFT-based versions pertaining to single crystal and polycrystalline substrates, and (iii) potentiality of Ising-type models in analysing two-dimensional phase transitions involving UPD adlayers, are emphasised.

The potential at which monolayer formation occurs, plays a crucial role in influencing the magnitude of the UPD shifts. Consequently, the choice of the adsorption isotherm(s) is inherently linked to the quantification of the UPD shifts. While diverse adsorption isotherms for ions and neutral organic compounds have been proposed ${ }^{84}$ in the case of metal/electrolyte interfaces, the precise transcription of these results to the UPD phenomena is still lacking. This issue is of paramount importance in so far as the correlation predicted by (1) pre-supposes a value for surface coverage for the depositing species. Furthermore, a precise theoretical estimate of electrosorption valency vis-a-vis partial charge transfer can arise only from a satisfactory adsorption isotherm. In contrast to the phenomenological Frumkin isotherm customarily employed in the context of electrosorption, it is more appropriate to employ isotherms which incorporate the specificity of ions, solvent dipoles and electrode surfaces, preferably within a quantum-mechanical formalism. This issue becomes crucial, especially since micrograms of adsorbed species can be routinely measured employing electrochemical quartz crystal microbalance (EQCM) studies, thereby warranting an accurate description of the electrosorption process. In fact, the isomorphism between cyclic voltammetry and EQCM frequencies has recently been demonstrated for UPD systems. ${ }^{85}$

Apart from the computational exercise involved in comprehending the UPD phenomenon, extensive studies concerning the electrocatalytic effects of UPD systems ranging from fuel cells and supercapacitors to hydrogen evolution and self-assembled monolayers are being pursued with diverse objectives. In view of this multi-faceted significance, among several frontier areas of interfacial electrochemistry, underpotential deposition and phase transitions involving them, occupy a prominent place.

\section{Acknowledgements}

We thank the reviewers for valuable suggestions on an earlier version of the manuscript. Financial support from the Council of Scientific and Industrial Research, Government of India is gratefully acknowledged.

\section{References}

1. Kolb D M, Prazasnyski M and Gerischer H $1974 J$. Electroanal. Chem. $\mathbf{5 4} 25$

2. Kolb D M 1978 Advances in electrochemistry and electrochemical engineering (eds) $\mathrm{H}$ Gerischer and C W Tobias (New York: John Wiley Interscience) vol. 11, p. 125

3. Aramata A 1997 Modern aspects of electrochemistry (eds) J O’M Bockris, R E White and B E Conway (New York: Plenum) vol. 31, p. 181

4. Herrero E, Buller L J and Abruna H D 2001 Chem. Rev. 1011897

5. Toney M and Melroy O R 1991 Electrochemical interfaces-Modern techniques for in situ characterization (ed) H D Abruna (Berlin: VCH) p. 57

6. Gewirth A A and Niece B K 1997 Chem. Rev. 97 1129

7. Kötz R 1990 Advances in electrochemical science and engineering (eds) $\mathrm{H}$ Gerischer $\mathrm{H}$ and $\mathrm{C} W$ Tobias (Weinheim: VCH) vol. 1, p. 75

8. Lehmpfuhl G, Uchida Y, Zei M S and Kolb D M 1999 Imaging of surfaces and interfaces (eds) J Lipkowski and P N Ross (New York: Wiley) p. 57

9. Leiva E 1996 Electrochim. Acta 412185

10. Blum L, Huckaby D A and Legault M D 1996 Electrochim. Acta 412207

11. Rikvold P A, Brown G, Novotny M A and Wieckowski A 1998 Coll. Surf. A 1343

12. Adzic R R 2002 Encyclopedia of electrochemistry (eds) A J Bard and M Stratmann (Weinheim: WileyVCH Verlag) vol. 1, p. 561 
13. Jennings G K and Laibinis P E 1997 J. Am. Chem. Soc. 1195208

14. Rikvold P A, Zhang J and Sung Y E 1996 Electrochim. Acta 412175

15. Brown G, Rikvold P A, Mitchell S J and Novotny M A 1999 Interfacial electrochemistry: Theory, experiment and applications (ed.) A Wieckowski (New York: Marcel Dekker) p. 47

16. Blum L, Legault M D and Huckaby D A 1999 Interfacial electrochemistry: Theory, experiment and applications (ed.) A Wieckowski (New York: Marcel Dekker) p. 19

17. Sanchez C and Leiva E 1999 Electrochim. Acta 45 691

18. Schmickler W 1999 Annual reports on the progress of chemistry vol. 95 Section $C$ (London: The Royal Society of Chemistry) p. 128

19. Sudha V and Sangaranarayanan M V 2002 J. Phys. Chem. B106 2699

20. Bockris J O'M and Reddy A K N 2000 Modern electrochemistry 2A 2nd edn (New York: Plenum) chapters 6 and 7

21. Trasatti S 1978 Advances in electrochemistry and electrochemical engineering (eds) $\mathrm{H}$ Gerischer and $\mathrm{C} \mathrm{W}$ Tobias (New York: John Wiley Interscience) vol. 10, p. 213

22. Bewick A and Thomas B 1976 J. Electroanal. Chem. 70239

23. Leiva E P M 1993 J. Electroanal. Chem. 3501

24. Takayanagi K, Kolb D M, Kambe K and Lehmpfuhl G 1980 Surf. Sci. 100407

25. Sudha V and Sangaranarayanan M V 2003 J. Phys. Chem. B107 3907

26. See for example, Conway B E, Gileadi E and Dzieciuch M 1963 Electrochim. Acta 8143

27. Angerstein-Kozlowska H, Macdougall B and Conway B E 1972 J. Electroanal. Chem. 39287

28. Gu R A, Cao P G, Sun Y H and Tian Z Q 2002 J. Electroanal. Chem. $\mathbf{5 2 8} 121$

29. Aurbach D and Gottlieb H 1989 Electrochim. Acta 34 141

30. Goren E, Chusid O and Aurbach D 1991 J. Electrochem. Soc. 138 L6

31. Badiali J P 1986 Electrochim. Acta 31149

32. Schmickler W 1990 Chem. Phys. 14195

33. Schmickler W 1996 Chem. Rev. 963177

34. Sanchez C and Leiva E 1998 J. Electroanal. Chem. 458183

35. Hohenberg P and Kohn W 1964 Phys. Rev. B136 864

36. Kohn W and Sham L J 1965 Phys. Rev. A140 1133

37. Smith J R 1969 Phys. Rev. 181522

38. Leiva E and Schmickler W 1995 Electrochim. Acta 4037

39. Saradha R and Sangaranarayanan M V 1998 J. Phys. Chem. B102 5468

40. Ashcroft N W 1966 Phys. Lett. 2348

41. Lehnert W and Schmickler W 1991 J. Electroanal. Chem. 31027

42. Roudgar A and Gross A 2003 J. Electroanal. Chem. 548121
43. Barbec V, Kim M H, Christian S D and Dryhurst G 1979 J. Electroanal. Chem. Interfacial Electrochem. 100111

44. Elliott C M and Murray R W 1976 Anal. Chem. 48 259

45. de Levie R 1988 Chem. Rev. 88599

46. Ising E 1925 Z. Phys. 31253

47. Rangarajan S K 1977 J. Electroanal. Chem. 8293

48. Sangaranarayanan M V and Rangarajan S K $1984 J$. Electroanal. Chem. 176119

49. Pushpalatha K and Sangaranarayanan M V 1997 J. Electroanal. Chem. 42539

50. Bosco E 1994 J. Electroanal. Chem. 379509

51. Denny R A and Sangaranarayanan M V 1995 Chem. Phys. Lett. 239131

52. Glauber R J 1963 J. Math. Phys. 4294

53. Kawasaki K 1966 Phys. Rev. 147224

54. Mitchell S J, Brown G and Rikvold P A 2000 J. Electroanal. Chem. 49368

55. Zhang J, Sung Y E, Rikvold P A and Wieckowski A 1996 J. Chem. Phys. 1045699

56. Brown G, Rikvold P A, Novotny M A and Wieckowski A 1999 J. Electrochem. Soc. 1461035

57. Gimenez M C, Del Popolo M G and Leiva E P M 1999 Electrochim. Acta 45699

58. Machado E and Buendia G M 2004 J. Magn. Magn. Mater. 272-276 249

59. Onsager L 1944 Phys. Rev. 65117

60. Ma S K 1976 Modern theory of critical phenomena (ed.) W A Benjamin (Reading, MA: Addison Wesley)

61. Ranjbar Sh and Parsafar G A 1999 J. Phys. Chem. B103 7514

62. Baker G A Jr and Graves-Morris P 1981 Encyclopedia of mathematics (ed.) G C Rota (Reading, MA: Addison Wesley) vol. 13

63. Blum L and Huckaby D A 1991 J. Chem. Phys. 94 6887

64. Blume M, Emery V J and Griffiths R B 1971 Phys. Rev. A4 1071

65. Tarasenko A A and Jastrabik L 1997 J. Electroanal. Chem. 440201

66. Sivardiere J and Lajzerowicz J 1975 Phys. Rev. A11 2101

67. Schultze J W and Dickertmann D 1976 Surf. Sci. 54 489

68. Rajendran L and Sangaranarayanan M V 1997 J. Phys. Chem. B101 4583

69. Arun Prasad M and Sangaranarayanan M V 2004 Electrochim. Acta 49445

70. Rama Kant and Rangarajan S K 1990 J. Electroanal. Chem. 27719

71. Huckaby D A and Blum L 1991 J. Electroanal. Chem. 315255

72. Tanaka T 2002 Methods of statistical physics (Cambridge: University Press)

73. Fowler R and Guggenheim E A 1965 Statistical thermodynamics (Cambridge: University Press)

74. Kikuchi R 1951 Phys. Rev. 81988

75. Saradha R and Sangaranarayanan M V 1997 Langmuir 135470 
76. Guidelli R 1981 J. Electroanal. Chem. 12359

77. Schmickler W 1983 J. Electroanal. Chem. 14915

78. Huckaby D A, Legault M D and Blum L 1998 J. Chem. Phys. 1093600

79. Samant M G, Borges G L, Gordon J G, Melroy O R and Blum L 1987 J. Am. Chem. Soc. 1095970

80. Tadjeddine A, Guay D, Ladouceur M and Tourillon G 1991 Phys. Rev. Lett. 662235

81. Omar I H, Pauling H J and Juttner K 1993 J. Electrochem. Soc. 1402187
82. Inzelt G and Horanyi G 2000 J. Electroanal. Chem. 491111

83. Wandlowski T 2002 Encyclopedia of electrochemistry (eds) M Urbakh and E Gileadi (Weinheim: VCHWiley) vol. 1 , p. 383

84. Nikitas P 1996 Electrochim. Acta 412159

85. Girija T C and Sangaranarayanan M V 2005 J. Solid State Electrochem. (in press)

86. Pauling L 1960 The Nature of Chemical Bond 3rd edn (New York: Cornell Univ. Press) chapter 3 